\title{
Dynamical mean-field theory for light-fermion-heavy-boson mixtures on optical lattices
}

\author{
M. Iskin ${ }^{1}$ and J. K. Freericks ${ }^{2}$ \\ ${ }^{1}$ Department of Physics, Koç University, Rumelifeneri Yolu, 34450 Sariyer, Istanbul, Turkey \\ ${ }^{2}$ Department of Physics, Georgetown University, Washington, D.C. 20057, USA
}

(Received 1 September 2009; published 24 November 2009)

\begin{abstract}
We theoretically analyze Fermi-Bose mixtures consisting of light fermions and heavy bosons that are loaded into optical lattices (ignoring the trapping potential). To describe such mixtures, we consider the Fermi-Bose version of the Falicov-Kimball model on a periodic lattice. This model can be exactly mapped onto the spinless Fermi-Fermi Falicov-Kimball model at zero temperature for all parameter space as long as the mixture is thermodynamically stable. We employ dynamical mean-field theory to investigate the evolution of the FermiBose Falicov-Kimball model at higher temperatures. We calculate spectral moment sum rules for the retarded Green's function and self-energy, and use them to benchmark the accuracy of our numerical calculations, as well as to reduce the computational cost by exactly including the tails of infinite summations or products. We show how the occupancy of the bosons, single-particle many-body density of states for the fermions, momentum distribution, and the average kinetic energy evolve with temperature. We end by briefly discussing how to experimentally realize the Fermi-Bose Falicov-Kimball model in ultracold atomic systems.
\end{abstract}

DOI: $10.1103 /$ PhysRevA.80.053623

PACS number(s): 03.75.Lm, 37.10.Jk, 67.85.Pq

\section{INTRODUCTION}

Experimental work in ultracold atomic systems in optical lattices has been progressing rapidly. First generation experiments focused primarily on single species systems, or mixtures of different isotopes of the same atomic species. Now, experimental work is progressing into the realm of mixtures of different species of atoms. Since these atomic species often have significantly different masses, one immediately expects such systems to respond differently than isotopic mixtures. In addition, if light alkali-metal atoms such as $\mathrm{Li}$ or $\mathrm{K}$ are used, or if one uses alkaline-earth-metal atoms such as $\mathrm{Sr}$ or rare-earth metals such as $\mathrm{Yb}$, then one has the prospect for modifying the particle statistics from Fermi-Dirac to Bose by simply changing the isotope employed in the experiment.

In this work, we focus on Fermi-Bose mixtures where the bosonic atoms are the heavy atoms (which is typically the experimental situation if $\mathrm{Li}$ or $\mathrm{K}$ is the light fermion and $\mathrm{Rb}$, $\mathrm{Cs}, \mathrm{Sr}$, or $\mathrm{Yb}$ is the heavy boson). While much work on Fermi-Bose mixtures has focused on either how the presence of the fermions modifies the Bose-Einstein condensation (BEC) of the bosons, or how the presence of the bosons modifies the interactions of the fermions (e.g., by allowing phonons) [1], our work here focuses on the opposite situation where the heavy bosons are so heavy that we can ignore the quantum-mechanical effects of their kinetic energy, and hence they never condense into a BEC. Instead, at low temperature, the mixture either phase separates or forms static density wave patterns, which can be quite complex. We believe such a situation arises when the tunneling amplitude of the heavy bosons on the optical lattice is more than an order of magnitude smaller than the tunneling amplitude of the light fermions. This typically occurs when the lattice depth is deep (more than 10 to 15 recoil energies of the $\mathrm{Rb}$ for a $\mathrm{K}-\mathrm{Rb}$ mixture) and the system is well represented by a single-band model [2]. In this situation, experiments are likely to be run at temperatures significantly higher than the BEC temperature, and it is more likely that static density waves would form instead of superfluidity (in other words, we are examining how the fermions modify the Mottinsulating state of the bosons, which occurs when the bosonboson repulsion is much larger than the boson tunneling amplitude [3]). Long-range effective boson-boson interactions are generated via the interactions with the mobile fermions. This realm is not so well known within the atomic physics community, although similar models have been widely studied within the condensed-matter physics community, as we describe below.

Recently, mixtures of fermionic ${ }^{40} \mathrm{~K}$ and bosonic ${ }^{87} \mathrm{Rb}$ atoms have been first studied at fixed interspecies interaction strengths by two different experimental groups [4,5], and later with tunable interactions [6], where a shift of the bosonic superfluid to Mott insulator transition has been observed due to the interspecies coupling (irrespective of its sign but with significant asymmetry). There are several theoretical proposals to explain this effect [7-11]. Motivated by these experiments, here we study such light-Fermi-heavyBose mixtures with the Fermi-Bose version of the FalicovKimball (FK) model [14-16], the Fermi-Fermi version of which has been widely discussed in the condensed-matter literature $[17,18]$. In our case, the bosons have no quantum dynamics of motion, but they can sample all possible heavyatom configurations in an annealed statistical-mechanical sense.

Our main results are as follows. First, we examine the symmetries of the Hamiltonian, and show that the FermiBose FK model can be mapped exactly onto the spinless Fermi-Fermi FK model at zero temperature for all parameter space as long as the mixture is thermodynamically stable. Since this mapping is only approximate at low temperatures and it fails at high temperatures, we develop dynamical mean-field theory (DMFT) [19] (which becomes exact in infinite dimensions) to investigate the effects of temperature and how the Fermi-Bose system evolves into an effective Fermi-Fermi system. In addition, we calculate spectral moment sum rules for the retarded Green's function and self- 
energy, and use them to check the accuracy of our numerical calculations, as well as to reduce the computational cost [20-23]. We also present typical numerical results for the Fermi-Bose FK model including the occupancy of the bosons, single-particle many-body density of states (DOS) for the fermions, momentum distribution, and the average kinetic energy. The DOS has significant temperature dependence as the system evolves from high to low temperatures. For example, in the insulating regime, where the magnitude of the boson-fermion interaction is much larger than the magnitude of the fermion tunneling amplitude, many "upper Hubbard bands" exist at high temperature, with the weights of each band determined by the probability that the bosons multiply occupy a lattice site. These bands then evolve to just one upper and one lower Hubbard band at low temperature.

The remainder of this manuscript is organized as follows. In Sec. II, we first introduce the Hamiltonian for the FermiBose version of the FK model, and then discuss its symmetries. In Sec. III, we develop the DMFT for this model, where we also discuss spectral moment sum rules for the retarded Green's function and self-energy. The numerical results obtained from the DMFT are discussed in Sec. IV, and a brief summary and conclusions are presented in Sec. V.

\section{LIGHT FERMION-HEAVY BOSON MIXTURES}

We analyze Fermi-Bose mixtures consisting of light fermions and heavy bosons that are loaded into optical lattices. We consider the limiting case such that the band mass of bosons $M_{b}$ is much greater than that of fermions $\left(M_{b} \gg M_{f}\right)$. In this case, the tunneling term for the bosons $t_{b}$ is much smaller than that of the fermions $\left(t_{b} \ll t_{f}\right)$, and we may set $t_{b}=0$ in the quantum-mechanical Hamiltonian that describes the system [12]. Such mixtures could be experimentally realized in ultracold atomic systems. For instance, a ${ }^{40} \mathrm{~K}-{ }^{87} \mathrm{Rb}$ mixture with $M_{b} \approx 2.2 M_{f}$, a ${ }^{6} \mathrm{Li}^{-}{ }^{41} \mathrm{~K}$ mixture with $M_{b}$ $\approx 6.8 M_{f}$, or a ${ }^{6} \mathrm{Li}-{ }^{133} \mathrm{Cs}$ mixture with $M_{b} \approx 22.2 M_{f}$ are good candidates for the applicability of this Hamiltonian [13]. Hence, even with a mass difference of 2 , one can get a significant difference in the hopping. In addition, one could also create species-dependent optical lattices for different isotopes of the same atom such that the bosonic isotope is localized but the fermionic one is not, which might be most relevant for isotopic mixtures of $\mathrm{Sr}$ or $\mathrm{Yb}$ (in other words, it is not the masses per se that need to be different, but it is the tunneling amplitudes when in an optical lattice that need to be quite different). It is important to emphasize that, although we consider the limit of localized bosons, the problem at hand is still a strongly correlated many-body problem since the bosons and fermions are coupled by the interaction and we take annealed statistical averages over all heavy-atom configurations.

\section{A. Fermi-Bose Falicov-Kimball (FK) model}

With such systems in mind, we analyze the following Hamiltonian to describe the light-fermion-heavy-boson mixtures [14-16]

$$
\begin{aligned}
H= & -t_{f} \sum_{\langle i, j\rangle}\left(f_{i}^{\dagger} f_{j}+f_{j}^{\dagger} f_{i}\right)+\frac{U_{b b}}{2} \sum_{i} b_{i}^{\dagger} b_{i}\left(b_{i}^{\dagger} b_{i}-1\right) \\
& +U_{b f} \sum_{i} b_{i}^{\dagger} b_{i} f_{i}^{\dagger} f_{i}-\mu_{f} \sum_{i} f_{i}^{\dagger} f_{i}-\mu_{b} \sum_{i} b_{i}^{\dagger} b_{i},
\end{aligned}
$$

where $f_{i}^{\dagger}\left(f_{i}\right)$ is the creation (annihilation) operator for an itinerant spinless fermion at site $i$, and $b_{i}^{\dagger}\left(b_{i}\right)$ is the corresponding operator for a localized boson at site $i$. The fermionic operators satisfy the usual canonical anticommutation relations $\left\{f_{i}, f_{j}^{\dagger}\right\}=\delta_{i j}$ where $\delta_{i j}$ is the Kronecker delta function, the bosonic operators satisfy the usual canonical commutation relations $\left[b_{i}, b_{j}^{\dagger}\right]=\delta_{i j}$, and the fermionic and bosonic operators all mutually commute. The first term is the kinetic energy of the fermions with $t_{f}$ denoting the nearestneighbor tunneling. The second and third terms are the onsite density-density interactions between the bosons themselves, and between the bosons and fermions, respectively. The on-site fermion-fermion interaction is not allowed because of the Pauli exclusion principle, i.e., $U_{f f} \rightarrow \infty$. The last two terms involve the chemical potentials of the fermions $\left(\mu_{f}\right)$ and bosons $\left(\mu_{b}\right)$, that are employed to adjust the filling of the corresponding particles to the desired values.

The model Hamiltonian given in Eq. (1) then corresponds to the Fermi-Bose version of the FK model, the Fermi-Fermi version of which has been studied extensively in the condensed-matter literature $[17,18]$. At low temperatures, the Fermi-Fermi version of the model is known to possess significant regions of density wave order with complicated patterns [17], and also has a strong tendency to phase separate when the light-fermion-heavy-fermion interaction is large and repulsive [24]. It is also known that the heavy fermions cannot generate an effective retarded light-fermion-lightfermion attraction leading to superconductivity as a consequence of Anderson's theorem [25]. Within the DMFT context, essentially all response functions (both static and dynamical) and all kinds of transport have been evaluated for the system [18]. We show below that many of these low temperature properties are shared by the Fermi-Bose FK model, but at higher temperatures, the behavior is quite different.

\section{B. Symmetries of the Hamiltonian}

This Hamiltonian possesses partial particle-hole symmetries for the fermions and bosons. These symmetries hold on a bipartite lattice where it is possible to divide the entire lattice into two sublattices $A$ and $B$ such that the fermionic tunneling only connects different sublattices. When the particle-hole transformation is applied to the fermions, i.e., $f_{i}^{\dagger} \rightarrow f_{i}^{h}(-1)^{p(i)}$ and $f_{i} \rightarrow\left(f_{i}^{h}\right)^{\dagger}(-1)^{p(i)}$ with $p(i)=0$ for $i \in A$ and $p(i)=1$ for $i \in B$, then, up to a numerical shift, it can be shown that the Hamiltonian that is expressed in terms of the hole operators for the fermions maps onto the starting Hamiltonian with $\mu_{f} \rightarrow-\mu_{f}, U_{b f} \rightarrow-U_{b f}$, and $\mu_{b} \rightarrow \mu_{b}-U_{b f}$. In the canonical ensemble, this means that the energies are simply related by $E\left(\rho_{f}, \rho_{b}, U_{b f}\right) \equiv E\left(1-\rho_{f}, \rho_{b},-U_{b f}\right)$, where $\rho_{f}$ and $\rho_{b}$ are the fillings for fermions and bosons, respectively. We notice that this mapping for the fermions holds at any temperature $T$, unlike that for the bosons, discussed next. 
When the particle-hole transformation is applied to the bosons, i.e., $b_{i}^{\dagger} \rightarrow b_{i}^{h}(-1)^{p(i)}$ and $b_{i} \rightarrow\left(b_{i}^{h}\right)^{\dagger}(-1)^{p(i)}$, then, up to a numerical shift, it can also be shown that the Hamiltonian that is expressed in terms of the hole operators for the bosons maps onto the starting Hamiltonian with $\mu_{f} \rightarrow \mu_{f}+U_{b f}$ and $\mu_{b} \rightarrow \mu_{b}+U_{b b}$. In the canonical ensemble, this means that the energies of many-body eigenstates are simply related by $E\left(\rho_{f}, \rho_{b}, U_{b f}\right) \equiv E\left(\rho_{f}, 1+\rho_{b}, U_{b f}\right)$. This is not enough to map Green's functions onto each other. The subtle issue is that the particle states with $n_{b}=0,1,2, \ldots, \infty$ map onto the hole states $n_{b}^{h} \equiv n_{b}+1=1,2,3, \ldots, \infty$, hence we lose all information about the weight of the $n_{b}^{h}=0$ state when more than one $n_{b}$ $>0$ state has a nonzero density; when only two states are nonzero, the mapping above plus the relation between particle and hole densities allows us to find all of the bosonic weights. This occurs exactly only at $T=0$ where at most two states have nonzero weights. Hence the bosonic particle-hole symmetry only occurs at $T=0$. This symmetry also holds in the atomic $\left(t_{b}=0\right)$ limit of the Bose-Hubbard model at $T=0$, where the energy for the particle excitations of the $n_{b}$ th state is degenerate with that for the hole excitations of the $\left(n_{b}\right.$ $+1)$ th state, but it does not hold when $t_{b} \neq 0$, because one can no longer label the many-body eigenstates by the local boson particle number.

The latter symmetry implies that there can be at most two bosonic states that can be occupied at $T=0$. For instance, $n_{b}=\{0,1\}$ states for $0<\rho_{b}<1 ; n_{b}=1$ state for $\rho_{b}=1 ; n_{b}$ $=\{1,2\}$ states for $1<\rho_{b}<2 ; n_{b}=2$ state for $\rho_{b}=2 ; n_{b}$ $=\{2,3\}$ states for $2<\rho_{b}<3$; etc. This is similar to what happens in the spinless Fermi-Fermi FK model where there can be at most two fermionic states for the heavy fermions due to the Pauli exclusion principle. Therefore, the Fermi-Bose FK model can be exactly mapped onto the well-studied spinless Fermi-Fermi FK model for all parameter space at $T=0$ as long as the mixture is thermodynamically stable. Since this mapping is only approximate at low temperatures, and it fails at high temperatures, we develop DMFT to investigate the effects of temperature.

\section{DYNAMICAL MEAN-FIELD THEORY}

The DMFT for the Fermi-Fermi FK model is well established in the literature $[18,19]$, and it can be easily generalized to the Fermi-Bose case that we consider here. For this purpose, we notice that the bosons cannot undergo BEC since there are a fixed number of localized bosons on each lattice site and the local boson particle number is an operator that commutes with the Hamiltonian. Then the impurity partition function can be integrated analytically since the effective action is quadratic in the fermionic operators and depends only on the local bosonic number operators. As a result, similar to the Fermi-Fermi case, there are three main equations that need to be solved self-consistently for the Fermi-Bose FK model.

\section{A. Imaginary-axis formalism}

The first equation is the single-particle lattice Green's function for the fermions defined by the time-ordered prod- uct $G(\tau)=-\left\langle T_{\tau} f_{i}(\tau) f_{i}^{\dagger}(0)\right\rangle$ in the imaginary time $(0 \leq \tau \leq \beta)$ representation, where $T_{\tau}$ is the imaginary time ordering operator, and $\langle O\rangle \equiv \operatorname{Tr}\left\{e^{-\beta H} O\right\} / Z$ is the ensemble average with $Z=\operatorname{Tr}\left\{e^{-\beta H}\right\}$ the partition function and $\beta=1 / T$ the inverse temperature. Here, the creation and annihilation operators are in the imaginary-time Heisenberg representation $O(\tau)$ $=e^{H \tau} O(0) e^{-H \tau}$. In the Matsubara frequency representation, $G\left(i \omega_{n}\right)=\int_{0}^{\beta} d \tau e^{i \omega_{n} \tau} G(\tau)$, where $\omega_{n}=(2 n+1) \pi / \beta$ is the fermionic Matsubara frequency, the Green's function becomes

$$
G\left(i \omega_{n}\right)=\sum_{n_{b}=0}^{\infty} \frac{w_{n_{b}}}{G_{0}^{-1}\left(i \omega_{n}\right)-U_{b f} n_{b}},
$$

where $w_{n_{b}}=Z_{n_{b}} / Z$ (with $Z=\sum_{n_{b}=0}^{\infty} Z_{n_{b}}$ ) is the probability for each site to be occupied exactly by $n_{b}=0,1,2, \ldots, \infty$ bosons, and $G_{0}^{-1}\left(i \omega_{n}\right)=i \omega_{n}+\mu_{f}-\lambda\left(i \omega_{n}\right)$ is the bare Green's function with $\lambda\left(i \omega_{n}\right)$ the dynamical mean field. Here,

$$
Z_{n_{b}}=e^{\beta\left[\mu_{b} n_{b}-U_{b b} n_{b}\left(n_{b}-1\right) / 2\right]} Z_{0}\left(\mu_{f}-U_{b f} n_{b}\right)
$$

is the partition function for the $n_{b}$ state, where

$$
Z_{0}\left(\mu_{f}\right)=2 e^{\beta \mu_{f} / 2} \prod_{n=-\infty}^{\infty} \frac{i \omega_{n}+\mu_{f}-\lambda\left(i \omega_{n}\right)}{i \omega_{n}}
$$

is the fermionic partition function for $U_{b f}=0$. The prefactor in Eq. (4) is added to give the correct noninteracting result when $\lambda\left(i \omega_{n}\right)=0$. In the case of the spinless Fermi-Fermi FK model, due to the Pauli exclusion principle, i.e., the heavy fermion-heavy fermion interaction $U_{b b} \rightarrow \infty$, only two states $\left(w_{0}\right.$ and $\left.w_{1}\right)$ can be occupied such that $w_{1}=1-w_{0}$ is the filling of localized fermions. Note these equations are similar to the solution of the classical Holstein model [26], but here the boson states are discrete, while there they are continuous.

The partition functions also satisfy the well-known relations $Z_{0}\left(\mu_{f}\right)=\operatorname{Det} G_{0}^{-1}\left(i \omega_{n}\right)$ and $Z=\operatorname{Det} G^{-1}\left(i \omega_{n}\right)$, so that the bare Green's function can be re-expressed as

$$
G_{0}^{-1}\left(i \omega_{n}\right)=G^{-1}\left(i \omega_{n}\right)+\Sigma^{-1}\left(i \omega_{n}\right),
$$

which is our second equation. This is Dyson's equation which relates the bare Green's function to the self-energy $\Sigma\left(i \omega_{n}\right)$, and can also be thought as the definition of the selfenergy.

The third equation is given by the lattice Hilbert transform of the noninteracting DOS,

$$
G\left(i \omega_{n}\right)=\int_{-\infty}^{\infty} \frac{d \epsilon \rho(\epsilon)}{i \omega_{n}+\mu_{f}-\Sigma\left(i \omega_{n}\right)-\epsilon},
$$

where $G\left(i \omega_{n}\right)=\Sigma_{\mathbf{k}} G\left(\mathbf{k}, i \omega_{n}\right) \quad$ with $\quad G\left(\mathbf{k}, i \omega_{n}\right)=1 /\left[i \omega_{n}+\mu_{f}\right.$ $\left.-\Sigma\left(i \omega_{n}\right)-\epsilon(\mathbf{k})\right]$ the momentum-resolved Green's function, and $\rho(\epsilon)=\Sigma_{\mathbf{k}} \delta[\epsilon-\epsilon(\mathbf{k})]=e^{-\left(\epsilon / t^{*}\right)^{2}} /\left(\sqrt{\pi} t^{*}\right)$ is the noninteracting DOS for the infinite-dimensional hypercubic lattice with $\delta(x)$ the delta function and $\boldsymbol{\epsilon}(\mathbf{k})=-2 t_{f} \sum_{i=1}^{d} \cos \left(k_{i} a\right)$ the energy dispersion for the fermions. Here, we used the fact that the tunneling $t_{f}$ scales with dimension $d$ such that $t_{f}=t^{*} / \sqrt{4 d}$ [27]. This equation can be rewritten in terms of the Faddeeva function $\quad G\left(i \omega_{n}\right)=-i \sqrt{\pi} e^{-\left[i \omega_{n}+\mu_{f}-\Sigma\left(i \omega_{n}\right)\right]^{2}} \operatorname{erfc}\left\{-i \eta\left[i \omega_{n}+\mu_{f}\right.\right.$ $\left.\left.-\Sigma\left(i \omega_{n}\right)\right]\right\}$, where $\operatorname{erfc}(z)=(2 / \sqrt{\pi}) \int_{z}^{\infty} d z e^{-z^{2}}$ is the complex 
complementary error function, and $\eta=\operatorname{Sign}\left\{\operatorname{Im}\left[i \omega_{n}\right.\right.$ $\left.\left.-\Sigma\left(i \omega_{n}\right)\right]\right\}$.

It is convenient to solve Eqs. (2), (5), and (6) selfconsistently by using an iterative approach [28]. For a fixed set of $U_{b b}, U_{b f}$, and $T$, our strategy is as follows: (1) choose initial values for $\mu_{f}$ and $\mu_{b}$; (2) start with an initial value for $\Sigma\left(i \omega_{n}\right)$, e.g., $\Sigma\left(i \omega_{n}\right)=0$, and plug it into Eq. (6) to solve for $G\left(i \omega_{n}\right)$; (3) use $G\left(i \omega_{n}\right)$ and the initial $\Sigma\left(i \omega_{n}\right)$ in Eq. (5) to obtain $G_{0}\left(i \omega_{n}\right)$; (4) plug $G_{0}\left(i \omega_{n}\right)$ into Eq. (2) to solve for the new $G\left(i \omega_{n}\right)$; (5) use $G_{0}\left(i \omega_{n}\right)$ and the new $G\left(i \omega_{n}\right)$ in Eq. (5) to obtain a new $\Sigma\left(i \omega_{n}\right)$; and (6) iterate steps (2)-(5) with the new $\Sigma\left(i \omega_{n}\right)$ until the solution converges. Finally, we (7) adjust $\mu_{f}$ and $\mu_{b}$ until the desired $\rho_{f}=\left\langle f_{i}^{\dagger} f_{i}\right\rangle$ and $\rho_{b}=\left\langle b_{i}^{\dagger} b_{i}\right\rangle$ values are reached. Typically, the self-energy converges to eight decimal points in less than twenty iterations (in a few seconds) for each choice of $\mu_{f}$ and $\mu_{b}$, and the entire procedure takes less than a minute to obtain the particular values for $\rho_{f}$ and $\rho_{b}$.

\section{B. Real-axis formalism}

Once the chemical potentials $\mu_{f}$ and $\mu_{b}$, and the occupation probabilities $w_{n_{b}}$ are calculated from the imaginary-axis calculation, we can employ the analytic continuation of Eqs. (2), (5), and (6) with $i \omega_{n} \rightarrow \omega+i 0^{+}$, to calculate $G(\omega)$ and $\Sigma(\omega)$ on the real axis using a similar iterative approach. Typically, the convergence is slower on the real axis than on the imaginary one, especially near the correlation induced band edges which leads to a number of poles in the selfenergy when $U_{b f}$ is large enough. Therefore, it is important to verify the consistency between the imaginary- and realaxis calculations.

For instance, one of the stringent tests is the comparison of the Green's function that is calculated directly on the imaginary axis by the algorithm described above to the spectral representation for the imaginary-axis Green's function given by $G\left(i \omega_{n}\right)=-(1 / \pi) \operatorname{Im} \int_{-\infty}^{\infty} d \omega G(\omega) /\left(i \omega_{n}-\omega\right)$, with the Green's function generated by the real-axis code appearing in the integrand. We usually achieve more than four digits of accuracy between the two calculations. Another test is the comparison of the filling of the fermions that can be calculated directly on the imaginary axis via $\rho_{f}$ $=(1 / \beta) \sum_{n=-\infty}^{\infty} G\left(i \omega_{n}\right)$ to that found from the real axis with $\rho_{f}=-(1 / \pi) \int_{-\infty}^{\infty} d \omega F(\omega) \operatorname{Im} G(\omega)$, where $F(\omega)=1 /\left(1+e^{\beta \omega}\right)$ is the Fermi-Dirac distribution function.

One can also check the spectral moment sum rules for the retarded Green's function, and also for the retarded selfenergy [20-23]. These moments are integrals of powers of frequency multiplied by the corresponding spectral function and integrated over all frequency. For instance, the spectral moments for the Green's function are defined as $\mu_{m}^{R}=$ $-(1 / \pi) \int_{-\infty}^{\infty} d \omega \omega^{m} \operatorname{Im} G(\omega)$. It can also be shown that $\mu_{m}^{R}$ $=\left\langle\left\{\left[f_{i}, H\right]_{m}, f_{i}^{\dagger}\right\}\right\rangle$, where $\left\{O_{1}, O_{2}\right\}=O_{1} O_{2}+O_{2} O_{1}$ is the anticommutator, and $\left[\mathrm{O}_{1}, \mathrm{O}_{2}\right]_{m}$ is the multiple commutation operator such that $\left[\mathrm{O}_{1}, \mathrm{O}_{2}\right]_{1}=\left[\mathrm{O}_{1}, \mathrm{O}_{2}\right]=\mathrm{O}_{1} \mathrm{O}_{2}-\mathrm{O}_{2} \mathrm{O}_{1}$; $\left[\mathrm{O}_{1}, \mathrm{O}_{2}\right]_{2}=\left[\left[\mathrm{O}_{1}, \mathrm{O}_{2}\right], \mathrm{O}_{2}\right]$; etc. Evaluating the commutators is tedious but straightforward, and the results are

$$
\mu_{0}^{R}=1 \text {, }
$$

$$
\begin{gathered}
\mu_{1}^{R}=-\left(\mu_{f}-U_{b f} \rho_{b}\right), \\
\mu_{2}^{R}=\frac{t^{* 2}}{2}+\left(\mu_{f}-U_{b f} \rho_{b}\right)^{2}+U_{b f}^{2}\left(\left\langle b_{i}^{\dagger} b_{i} b_{i}^{\dagger} b_{i}\right\rangle-\rho_{b}^{2}\right), \\
\mu_{3}^{R}=-\frac{3 t^{* 2}}{2}\left(\mu_{f}-U_{b f} \rho_{b}\right)-\left(\mu_{f}-U_{b f} \rho_{b}\right)^{3}-3 \mu_{f} U_{b f}^{2} \\
\times\left(\left\langle b_{i}^{\dagger} b_{i} b_{i}^{\dagger} b_{i}\right\rangle-\rho_{b}^{2}\right)+U_{b f}^{3}\left(\left\langle b_{i}^{\dagger} b_{i} b_{i}^{\dagger} b_{i} b_{i}^{\dagger} b_{i}\right\rangle-\rho_{b}^{3}\right),
\end{gathered}
$$

where the operator averages $\left\langle b_{i}^{\dagger} b_{i} b_{i}^{\dagger} b_{i} \cdots\right\rangle$ can be easily calculated from the knowledge of $w_{n_{b}}$ as $\left\langle\left(b_{i}^{\dagger} b_{i}\right)^{k}\right\rangle=\sum_{n_{b}=0}^{\infty} w_{n_{b}} n_{b}^{k}$, and

$$
\rho_{b}=\sum_{n_{b}=0}^{\infty} w_{n_{b}} n_{b}
$$

is the filling of the bosons. Therefore, the accuracy of the calculations can be further checked by comparing the realaxis calculation of the moments $\mu_{m}^{R}$ by directly performing the integrations over the real frequency to the exact results given in Eqs. (7)-(10).

Similarly, the spectral moment sum rules for the retarded self-energy are defined as $C_{m}^{R}=-(1 / \pi) \int_{-\infty}^{\infty} d \omega \omega^{m} \operatorname{Im} \Sigma(\omega)$. One can also calculate these moments by using Dyson's equation, and after some significant algebra, the results are

$$
\begin{gathered}
C_{0}^{R}=U_{b f}^{2}\left(\left\langle b_{i}^{\dagger} b_{i} b_{i}^{\dagger} b_{i}\right\rangle-\rho_{b}^{2}\right), \\
C_{1}^{R}=-U_{b f}^{2}\left(\mu_{f}+2 U_{b f} \rho_{b}\right)\left(\left\langle b_{i}^{\dagger} b_{i} b_{i}^{\dagger} b_{i}\right\rangle-\rho_{b}^{2}\right) \\
+U_{b f}^{3}\left(\left\langle b_{i}^{\dagger} b_{i} b_{i}^{\dagger} b_{i} b_{i}^{\dagger} b_{i}\right\rangle-\rho_{b}^{3}\right) .
\end{gathered}
$$

In this way, the accuracy of the calculations can be again benchmarked by comparing the real-axis integration of the spectral moments $C_{m}^{R}$ with the exact values given in Eqs. (12) and (13). In addition, we would like to mention that the large frequency limit of the real-axis self-energy

$$
\Sigma(\omega \rightarrow \infty)=U_{b f} \rho_{b}
$$

provides another independent check of the numerics.

Typically, there are a number of poles in the self-energy near the correlation induced band edges when $U_{b f}$ is large enough, and in order to guarantee the accuracy of the sum rules that are calculated on the real axis by direct quadrature, the pole contributions have to be included by hand as described here. Using Eqs. (2) and (5) with $i \omega_{n} \rightarrow \omega+i 0^{+}$, we find that the locations $\omega_{p}$ of these poles are determined by the transcendental equation

$$
\sum_{n_{b}=0}^{\infty}\left[w_{n_{b}} \prod_{m_{b} \neq n_{b}}^{\infty}\left(\omega_{p}+\mu_{f}-U_{b f} m_{b}\right)\right]=0 .
$$

In a typical calculation, we restrict the bosonic occupancies to be less than some maximal number of multiple boson occupancy. Then the transcendental equation becomes a finite equation which is easy to solve directly. Using such a procedure, the self-energy can then be written as $\Sigma(\omega)$ $=\Sigma_{\text {reg }}(\omega)+\Sigma_{p} R_{p} /\left(\omega-\omega_{p}+i 0^{+}\right)$, where $\Sigma_{\text {reg }}(\omega)$ is the regular 
piece, $p$ sums over the finite number of poles that were found to solve the truncated equation, and $R_{p}$ is the residue of the pole $\omega_{p}$ that is calculated next (only poles with positive residue are included in the sum as described below). Since the pole contribution dominates the spectral functions near the poles, one can expand Eq. (6) with $i \omega_{n} \rightarrow \omega+i 0^{+}$in powers of $\omega+\mu_{f}-\Sigma(\omega)$, and obtain $G(\omega) \approx g_{1} /\left[\omega+\mu_{f}-\Sigma(\omega)\right]$ $+g_{3} /\left[\omega+\mu_{f}-\Sigma(\omega)\right]^{3}+\cdots$ where $g_{1}=\int_{-\infty}^{\infty} d \epsilon \rho(\epsilon)=1$ and $g_{3}$ $=\int_{-\infty}^{\infty} d \epsilon \rho(\epsilon) \epsilon^{2}=t^{* 2} / 2$. Inserting this expansion into Eq. (5) to obtain $\Sigma(\omega)$ near the poles, and after some algebra, leads to

$$
\begin{aligned}
& R_{p}=\frac{\sum_{n_{b}=0}^{\infty}\left(w_{n_{b}}-\delta_{0 n_{b}}\right) \prod_{m_{b} \neq n_{b}}^{\infty}\left(\omega_{p}+\mu_{f}-U_{b f} m_{b}\right)}{\left(\omega_{p}+\mu_{f}\right)^{-1} \prod_{q \neq p}\left(\omega_{p}-\omega_{q}\right)} \\
& -\frac{\sum_{n_{b}=0}^{\infty} w_{n_{b}} \sum_{n_{b}^{\prime} \neq n_{b}}^{\infty} \prod_{m_{b} \neq\left\{n_{b}, n_{b}^{\prime}\right\}}^{\infty}\left(\omega_{p}+\mu_{f}-U_{b f} m_{b}\right)}{g_{3}^{-1} \prod_{q \neq p}\left(\omega_{p}-\omega_{q}\right)},
\end{aligned}
$$

where $\delta_{i j}$ is the Kronecker delta function. Only poles where the residue $R_{p}$ is positive correspond to real physical poles. As a result, the pole contribution to the self-energy moment $C_{m}^{R}$ is $\Sigma_{p^{\prime}} R_{p^{\prime}} \omega_{p^{\prime}}^{m}$, where $p^{\prime}$ sums over the poles with positive residues.

We recall that the spectral moment sum rules given in Eqs. (7)-(10) and Eqs. (12) and (13) have exactly the same form in the case of the Fermi-Fermi FK model [21-23]. However, in that case $\left\langle\left(b_{i}^{\dagger} b_{i}\right)^{k}\right\rangle=\left\langle b_{i}^{\dagger} b_{i}\right\rangle=w_{1}$ for any $k$ due to the fermionic anticommutators. In addition, since $w_{n_{b} \geq 2}=0$, the pole equation given in Eq. (15) reduces considerably leading to a single pole at $\omega_{1}=-\mu_{f}+U_{b f}\left(1-w_{1}\right)$, for which the residue equation given in Eq. (16) reduces to the wellknown result $R_{1}=w_{1}\left(1-w_{1}\right) U_{b f}^{2}-g_{3}$ [29].

\section{Asymptotic behavior for large frequencies}

There is another important application of the spectral moment sum rules given above. They can be used to evaluate the high-frequency asymptotic behavior of the Green's function, self-energy, and dynamical mean-field exactly. Therefore, this knowledge can be used to reduce the number of Matsubara frequencies that is needed to solve the imaginaryaxis equations [23].

When the Matsubara frequency is high enough, it can be shown that the Green's function can be written as $G\left(i \omega_{n}\right)$ $=\sum_{m=0}^{\infty} \mu_{m}^{R} /\left(i \omega_{n}\right)^{m+1}$, and similarly the self-energy can be written as $\Sigma\left(i \omega_{n}\right)=\Sigma(\infty)+\sum_{m=0}^{\infty} C_{m}^{R} /\left(i \omega_{n}\right)^{m+1}$, where $\Sigma(\infty)$ is a real constant, and it is the large-frequency limit of the selfenergy. These expansions follow from the definition of the spectral moment sum rules and the spectral formula for the retarded Green's function and self-energy. Inserting these expansions into Eq. (5), and using the definition of $G_{0}^{-1}\left(i \omega_{n}\right)$ given below Eq. (2), we obtain the asymptotic expansion of the dynamical mean-field as $\lambda\left(i \omega_{n}\right)=t^{* 2} /\left[2\left(i \omega_{n}\right)\right]-t^{* 2}\left(\mu_{f}\right.$ $\left.-U_{b f} \rho_{b}\right) /\left[2\left(i \omega_{n}\right)^{2}\right]+\cdots$. This asymptotic expansion along with the expansion above allow us to treat the highfrequency tails of some quantities as described next.
Let us take an energy cutoff $\epsilon_{c}$ which is much larger than the bandwidth of the interacting DOS, and use the asymptotic expansion to sum over the Matsubara frequencies that are higher than $\epsilon_{c}$. This also defines a cutoff $n_{c}$ for the Matsubara frequencies, given by the closest one to $\epsilon_{c}$ but lying below it, i.e., $\omega_{n_{c}}=\left(2 n_{c}+1\right) \pi / \beta \lesssim \epsilon_{c}$. Inserting the asymptotic expansions into Eq. (3), we obtain

$$
\begin{aligned}
Z_{n_{b}}= & 2 \exp \left\{\beta\left[\frac{\mu_{f}-U_{b f} n_{b}}{2}+\mu_{b} n_{b}-\frac{U_{b b}}{2} n_{b}\left(n_{b}-1\right)\right]\right\} \\
& \times \prod_{n=0}^{n_{c}}\left|\frac{G_{0}^{-1}\left(i \omega_{n}\right)-U_{b f} n_{b}}{i \omega_{n}}\right|^{2} \prod_{n=n_{c}+1}^{\infty} \mid 1+\frac{\mu_{f}-U_{b f} n_{b}}{i \omega_{n}} \\
& -\frac{t^{* 2}}{2\left(i \omega_{n}\right)^{2}}+\left.\frac{t^{* 2}\left(\mu_{f}-U_{b f} \rho_{b}\right)}{2\left(i \omega_{n}\right)^{3}}\right|^{2} .
\end{aligned}
$$

Here, since the complex conjugate of $i \omega_{n}$ is equal to $i \omega_{-n-1}$, we take the negative Matsubara frequencies into account by writing the absolute value squares in the products. To approximate the infinite products that have an infinite number of terms, we first rewrite the infinite products as the exponential of the sum of the logarithm of the individual terms. Then, for temperatures much lower than the bandwidth of the interacting DOS, we replace the sum by an integral, and then convert the integral over frequency to an integral over $z=1 / \omega$, leading to

$$
\begin{aligned}
& \prod_{n=n_{c}+1}^{\infty}\left|1+\frac{a}{i \omega_{n}}+\frac{b}{\left(i \omega_{n}\right)^{2}}+\frac{c}{\left(i \omega_{n}\right)^{3}}\right|^{2} \\
& \quad \approx \exp \left\{\frac{\beta}{2 \pi} \times \int_{0}^{1 / \epsilon_{c}} \frac{d z}{z^{2}} \ln \left[\left(1+b z^{2}\right)^{2}+z^{2}\left(a-c z^{2}\right)^{2}\right]\right\} .
\end{aligned}
$$

Here, $a=\mu_{f}-U_{b f} n_{b}, b=-t^{* 2} / 2$, and $c=t^{* 2}\left(\mu_{f}-U_{b f} \rho_{b}\right) / 2$. As a result, the use of asymptotic expressions for the Green's function, self-energy, and the dynamical mean field allow us to reduce the computational effort considerably for the imaginary-axis calculation by keeping $\epsilon_{c}$ small. Because the DOS extends farther out in energy as $T$ rises, one needs to increase $\epsilon_{c}$ in order to achieve the same level of accuracy for high $T$.

The asymptotic expansions can also be used to treat the tails in the summation for the fermion filling, i.e., $\rho_{f}$ $=(1 / \beta) \sum_{n=-\infty}^{\infty} G\left(i \omega_{n}\right)$, which leads to

$$
\begin{aligned}
\rho_{f}= & \frac{\mu_{0}^{R}}{2}-\frac{\beta \mu_{1}^{R}}{4}+\frac{\beta^{3} \mu_{3}^{R}}{48} \\
& +\frac{1}{\beta} \sum_{n=-n_{c}}^{n_{c}-1}\left[G\left(i \omega_{n}\right)-\frac{\mu_{0}^{R}}{i \omega_{n}}-\frac{\mu_{1}^{R}}{\left(i \omega_{n}\right)^{2}}-\frac{\mu_{2}^{R}}{\left(i \omega_{n}\right)^{3}}-\frac{\mu_{3}^{R}}{\left(i \omega_{n}\right)^{4}}\right] .
\end{aligned}
$$

Here, we use the standard representation of the Fermi-Dirac distribution function $F(x)=(1 / \beta) \sum_{n=-\infty}^{\infty} 1 /\left(i \omega_{n}-x\right)$ and its derivatives with respect to $x$ when $x \rightarrow 0$, to evaluate the coefficients of the $(1 / \beta) \sum_{n=-\infty}^{\infty} 1 /\left(i \omega_{n}\right)^{k}$ type. Since the contribution from the summation (which needs to be evaluated 

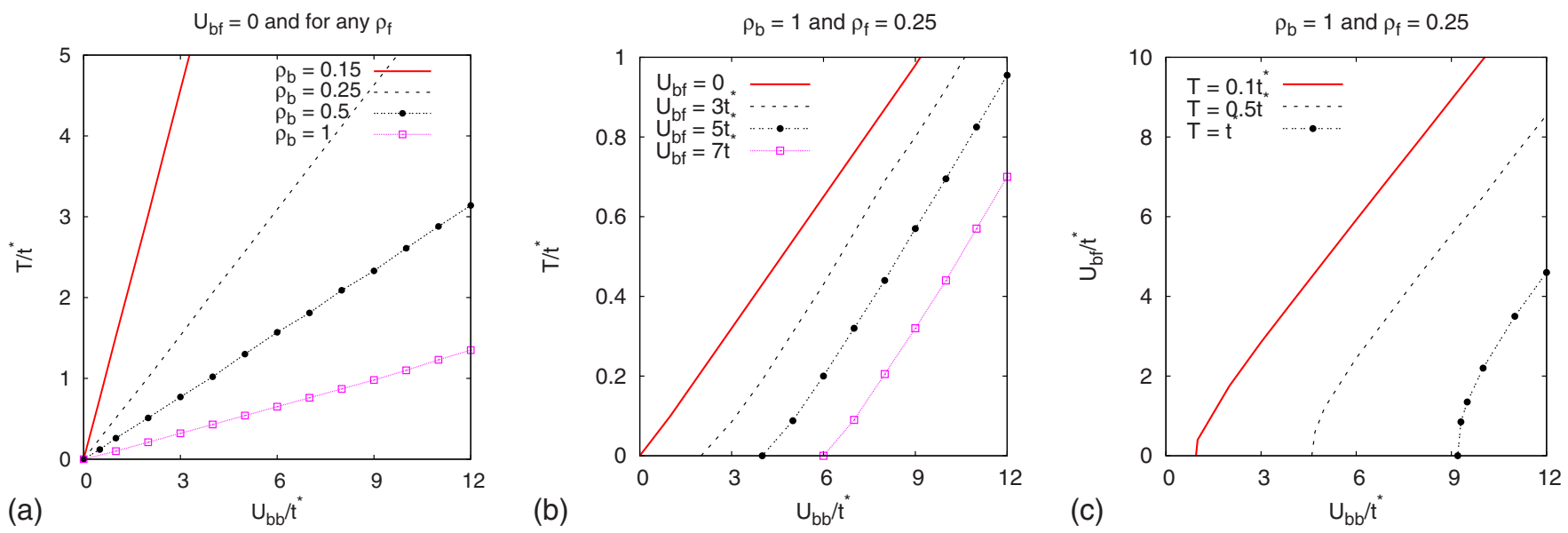

FIG. 1. (Color online) Mapping diagrams show when the Fermi-Bose FK model can be mapped onto the spinless Fermi-Fermi FK model as a function of boson-boson repulsion $U_{b b}$. In all figures, mapping boundaries separate the regions where total probability of finding a boson in two of the $n_{b}$ states is higher than $99 \%$ (which occurs below each line).

numerically) vanishes rapidly for large $\omega_{n}$, the filling can be calculated quite accurately.

\section{NUMERICAL RESULTS}

Having described the DMFT formalism for the FermiBose FK model, next we present some of our numerical results that are obtained by solving Eqs. (2), (5), and (6) selfconsistently for given fermion and boson fillings, $\rho_{f}$ and $\rho_{b}$, respectively. For this purpose, we choose a large energy cutoff $\epsilon_{c}=200 t^{*}$, and treat the tails of the high-frequency summations using the formalism described above. Since the system is unstable for $U_{b b}<0$, and the $U_{b f}<0$ case can be mapped onto the $U_{b f}>0$ case as discussed in Sec. II B, we restrict our analysis to $\left\{U_{b b}, U_{b f}\right\}>0$ values. We remark here that numerical solutions with $w_{n_{b} \rightarrow \infty} \neq 0$ are unphysical, indicating an instability in the system corresponding to a bosonic collapse when $U_{b b}<0$.

\section{A. Mapping onto the spinless Fermi-Fermi FK model}

We argued in Sec. II B that the Fermi-Bose FK model can be exactly mapped onto the well-studied spinless FermiFermi FK model for all parameter space at $T=0$ as long as the mixture is thermodynamically stable. Here, we numerically show how the system evolves away from the FermiFermi behavior at higher temperatures.

In Fig. 1, we calculate the region where the total probability of finding a boson in just two of the $n_{b}$ states is higher than $99 \%$. Therefore, below each line in all figures, the system can be effectively mapped onto the spinless Fermi-Fermi FK model. In Fig. 1(a), we show temperature $T$ vs bosonboson repulsion $U_{b b}$ mapping diagram for different boson fillings $\rho_{b}$ when bosons and fermions are uncoupled, i.e., $U_{b f}=0$. As $\rho_{b}$ increases, it is seen that the mapping is possible only for lower $T$ values at a given $U_{b b}$, and is possible only for lower $U_{b b}$ values at a given $T$. This is because it is energetically more favorable to have an occupation of multiple $n_{b}$ states as a function of increasing $T$ and/or decreasing $U_{b b}$ due to the Bose distribution function.
When $U_{b f} \neq 0$, we show a $T$ vs $U_{b b}$ mapping diagram for different $U_{b f}$ in Fig. 1(b), and a $U_{b f}$ vs. $U_{b b}$ mapping diagram for different $T$ in Fig. 1(c). As $U_{b f}$ increases, it is seen that the mapping is possible for smaller and smaller parameter space compared to the $U_{b f}=0$ limit. This is because the coupling between bosons and fermions induces an attractive interaction between bosons such that the effective boson-boson repulsion $U_{b b}^{\text {eff }}$ decreases by some amount that is proportional to $U_{b f}^{2}$ to the lowest order in $U_{b f}$. Therefore, increasing $U_{b f}$ leads to an occupation of additional $n_{b}$ states as discussed next.

\section{B. Density of states for the fermions}

In this subsection, we present our numerical results for the probability $w_{n_{b}}$ of finding a boson in state $n_{b}$, and the singleparticle many-body density of states (DOS) for the fermions, to illustrate typical properties of the Fermi-Bose FK model. The probabilities are shown in Fig. 2(a) as a function of $T$ for the first five boson occupancies when $\rho_{b}=1, \rho_{f}=0.25, U_{b b}$ $=t^{*}$, and $U_{b f}=1.7 t^{*}$. It is seen that $w_{n_{b}}=\delta_{1 n_{b}}$ at $T=0$ which is due to $\rho_{b}=\sum_{n_{b}=0}^{\infty} w_{n_{b}} n_{b}$ with $\sum_{n_{b}=0}^{\infty} w_{n_{b}}=1$. However, the occupation of the $n_{b}=1$ state decreases at finite $T$, while that of higher and higher $n_{b}$ states become finite with increasing $T$.

The occupancy of multiple $n_{b}$ states has a strong effect on the many-body DOS for the fermions, i.e., $A(\omega)=$ $-(1 / \pi) \operatorname{Im} G\left(i \omega_{n} \rightarrow \omega+i 0^{+}\right)$, which is given by

$$
A(\omega)=-\frac{1}{\pi} \operatorname{Im} \int_{-\infty}^{\infty} \frac{d \epsilon \rho(\epsilon)}{\omega+\mu_{f}-\Sigma(\omega)-\epsilon+i 0^{+}},
$$

where $\rho(\epsilon)$ is the noninteracting DOS for the infinitedimensional hypercubic lattice defined below Eq. (6), and the infinitesimal is needed only when $\operatorname{Im} \Sigma(\omega)=0$. In Fig. 2(b), there is a single Gaussian peak at $T=0$ corresponding to $n_{b}=1$ (which actually becomes a noninteracting system), but there are three peaks at $T=0.1 t^{*}$ corresponding to $n_{b}=0,1$ and 2. In addition, two more peaks occur at $T=t^{*}$ corresponding to $n_{b}=3$ and 4 . This is again because it is energetically more favorable to have an occupation of multiple $n_{b}$ 

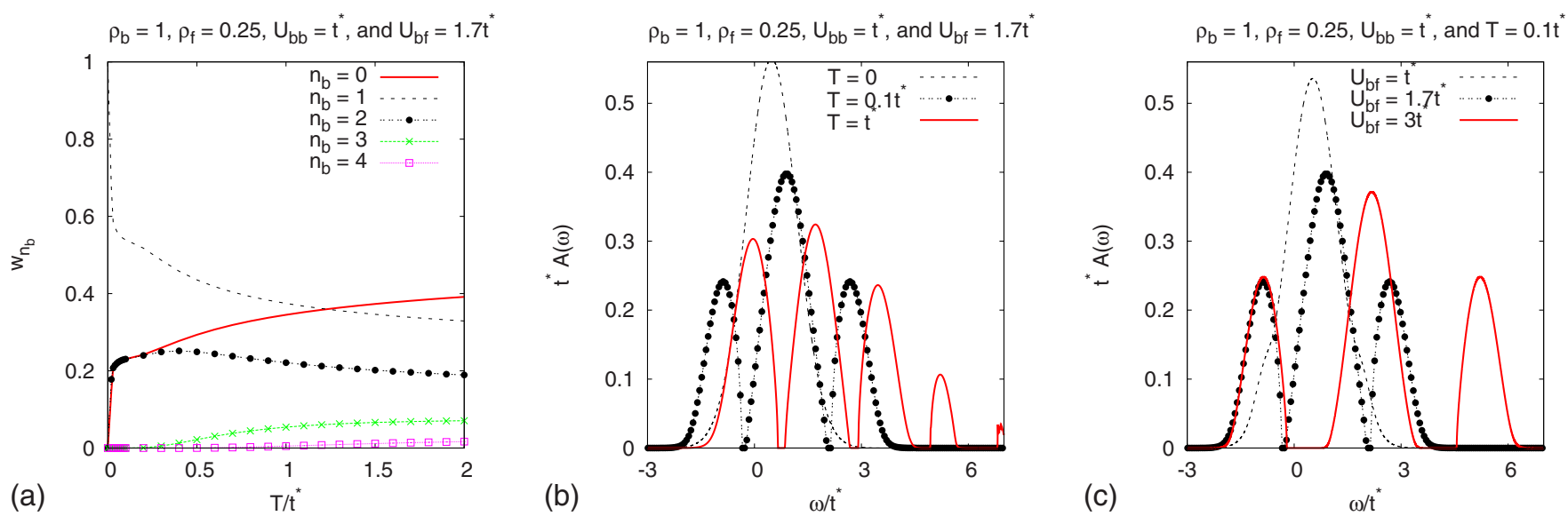

FIG. 2. (Color online) (a) The probability $w_{n_{b}}$ of finding a boson in state $n_{b}$ is shown as a function of temperature $T$ for the first five boson occupancies. [(b), (c)] The single-particle many-body density of states for the fermions $A(\omega)$ is shown as a function of frequency $\omega$.

states as a function of increasing $T$ due to the bosonic character of the heavy atoms. We mention that while there is not any pole in the self-energy when $T=0$, there are two physical poles at $\omega_{1} \approx-0.348 t^{*}$ and $\omega_{2} \approx 2.149 t^{*}$ with residues $R_{1}$ $\approx R_{2} \approx 0.166 t^{*}$ when $T=0.1 t^{*}$, and at $\omega_{1} \approx 0.725 t^{*}$ and $\omega_{2}$ $\approx 2.871 t^{*}$ with residues $R_{1} \approx 0.419 t^{*}$ and $R_{2} \approx 0.402 t^{*}$ when $T=t^{*}$. Notice that poles occur near the correlation induced band edges.

We investigate the effects of $U_{b f}$ on $A(\omega)$ in Fig. 2(c), where it is seen that increasing (decreasing) $U_{b f}$ increases (decreases) the number of peaks. This is again because the coupling between the bosons and fermions induces an attractive interaction between bosons such that the effective boson-boson repulsion decreases, which leads to an occupation of additional $n_{b}$ states. For instance, as shown in the figure, there is a single peak when $U_{b f}=t^{*}$, but there are three of them when $U_{b f}=1.7 t^{*}$. In addition, the band gap between different $n_{b}$ states increases with increasing $U_{b f}$. We again mention that while there is not any pole in the self-energy when $U_{b f}=t^{*}$, there are two physical poles at $\omega_{1} \approx-0.348 t^{*}$ and $\omega_{2} \approx 2.149 t^{*}$ with residues $R_{1} \approx R_{2} \approx 0.166 t^{*}$ when $U_{b f}$ $=1.7 t^{*}$, and at $\omega_{1} \approx 0.0605 t^{*}$ and $\omega_{2} \approx 4.295 t^{*}$ with residues $R_{1} \approx R_{2} \approx 1.759 t^{*}$ when $U_{b f}=3 t^{*}$. Notice again that poles occur near the correlation induced band edges.

Although the DOS for the fermions shows rich structures, this is not yet a measurable quantity in ultracold atomic systems (it might be feasible with an appropriately designed rf-frequency experiment that acts like a photoemission experiment of the fermions [30], but there one would be observing the DOS multiplied by the Fermi-Dirac distribution function, the so-called lesser spectral function). For this reason, we next discuss the momentum distribution of the fermions and bosons which could be easily measured in a timeof-flight measurement.

\section{Momentum distribution}

The occupancy of multiple $n_{b}$ states has also significant effect on the momentum distribution of the fermions, i.e., $n_{m d}(\mathbf{k})=(1 / \beta) \sum_{n=-\infty}^{\infty} G\left(\mathbf{k}, i \omega_{n}\right)$, which becomes

$$
n_{m d}(\mathbf{k})=\frac{1}{\beta} \sum_{n=-\infty}^{\infty} \frac{1}{i \omega_{n}+\mu_{f}-\Sigma\left(i \omega_{n}\right)-\epsilon(\mathbf{k})}
$$

where $G\left(\mathbf{k}, i \omega_{n}\right)$ is the momentum-resolved Green's function and $\boldsymbol{\epsilon}(\mathbf{k})=-2 t_{f} \sum_{i=1}^{d} \cos \left(k_{i} a\right)$ is the energy dispersion for the fermions defined below Eq. (6). The momentum distribution of the bosons is simply a constant given by the filling of the bosons since they are localized with zero tunneling. To regularize the frequency summation in our numerical calculations, we subtract $(1 / \beta) \sum_{n=-\infty}^{\infty} 1 /\left[i \omega_{n}+\mu_{f}-\Sigma(\infty)-\epsilon(\mathbf{k})\right]$, and add $F\left[-\mu_{f}+\Sigma(\infty)+\epsilon(\mathbf{k})\right]$ in Eq. (21), where $F(x)$ is the Fermi-Dirac distribution function.

Our numerical results for $n_{m d}(\mathbf{k})$ vs the fermion dispersion $\epsilon(\mathbf{k})$ are shown in Fig. 3. As shown in Fig. 3(a), $n_{m d}(\mathbf{k})$ is a step function at $T=0$, and it broadens as a function of increasing $T$, which is expected due to Fermi-Dirac statistics. For a fixed $T$, the effects of $U_{b f}$ on $n_{m d}(\mathbf{k})$ are shown in Fig. $3(\mathrm{~b})$, where it is seen that increasing $U_{b f}$ also broadens $n_{m d}(\mathbf{k})$ just like the temperature, which is the expected manybody effect due to a finite lifetime of the fermionic excitations. In the FK model, the fermions are usually not a Fermi liquid (or more correctly a Fermi gas in our context) at $T$ $=0$, but for the case of integer boson fillings $\rho_{b}$ $=0,1,2, \ldots, \infty$ (presented here) they are, because the system evolves to an effective noninteracting system as $T \rightarrow 0$.

\section{Average kinetic energy}

Another important quantity that can be measured in ultracold atomic systems is the average kinetic energy of the particles, which is given by $\langle\epsilon(\mathbf{k})\rangle=\Sigma_{\mathbf{k}} \epsilon(\mathbf{k}) n_{m d}(\mathbf{k})$ for the fermions, and vanishes for the bosons since they are localized with zero tunneling. Since the momentum distribution is measured directly in experiment, one can process the data to determine the average kinetic energy in the lattice under the assumption that the momentum distribution has not changed significantly during the time-of-flight experiment. For our calculations, we convert the summation over momentum to an integral over energy. The resulting expression is 

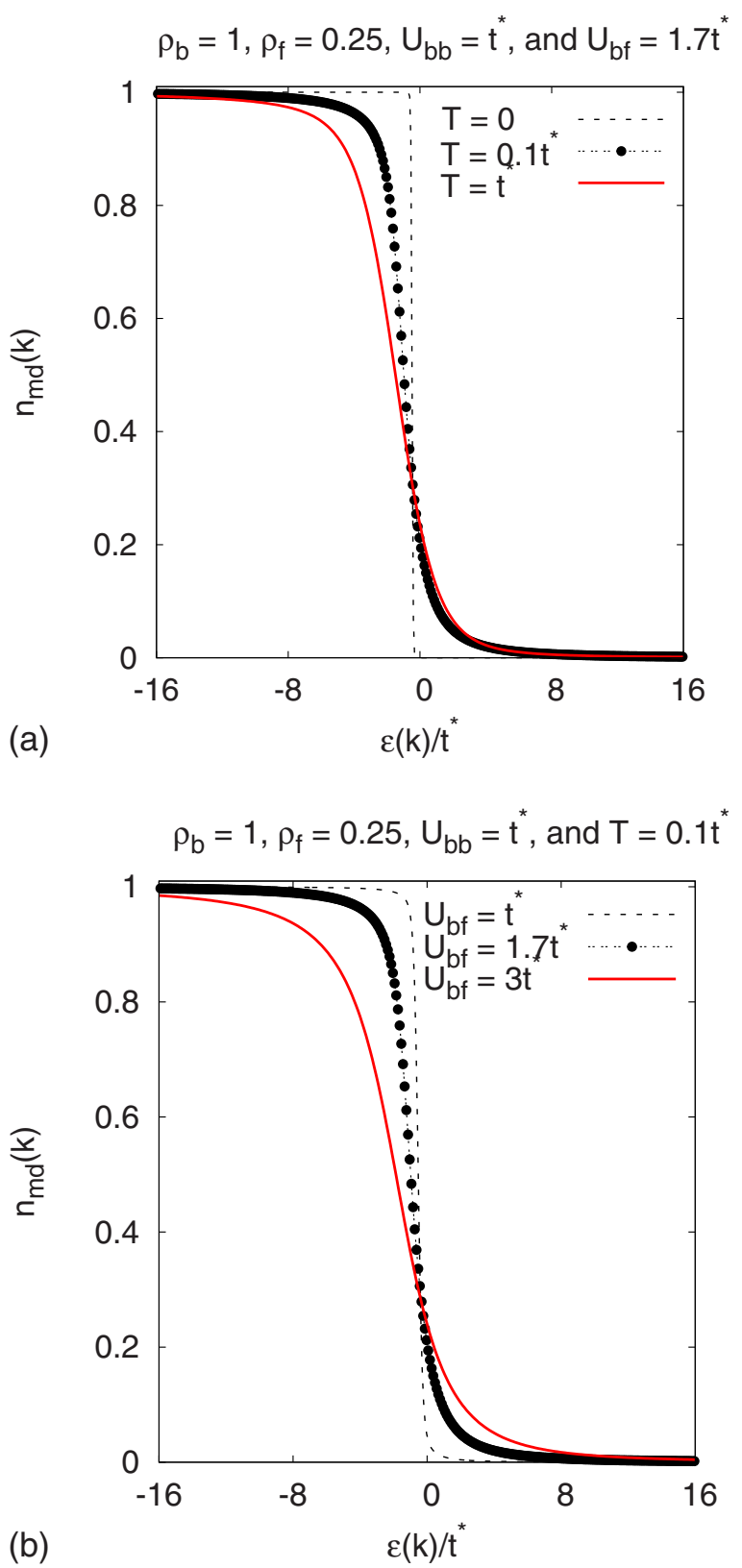

FIG. 3. (Color online) The momentum distribution of the fermions $n_{m d}(\mathbf{k})$ is shown as a function of the dispersion $\epsilon(\mathbf{k})$. The parameters used in these figures are the same as the ones used in Fig. 2.

$$
\langle\epsilon(\mathbf{k})\rangle=\int_{-\infty}^{\infty} d \epsilon \rho(\epsilon) \epsilon n_{m d}(\epsilon),
$$

where $\rho(\epsilon)$ is the noninteracting DOS for the infinitedimensional hypercubic lattice defined below Eq. (6).

Our numerical results for $\langle\epsilon(\mathbf{k})\rangle$ vs $T$ are shown in Fig. 4. When $U_{b f}$ is small, e.g., $U_{b f}=t^{*}$, it is seen that $\langle\epsilon(\mathbf{k})\rangle$ increases monotonically as a function of $T$. However, when $U_{b f}$ becomes large enough, see e.g., $U_{b f}=1.7 t^{*}$, it has a local minimum at finite $T$ after an initial increase for lower temperatures. This minimum moves toward higher $T$ as $U_{b f}$ becomes larger, compare, e.g., $U_{b f}=1.7 t^{*}$ case with that of

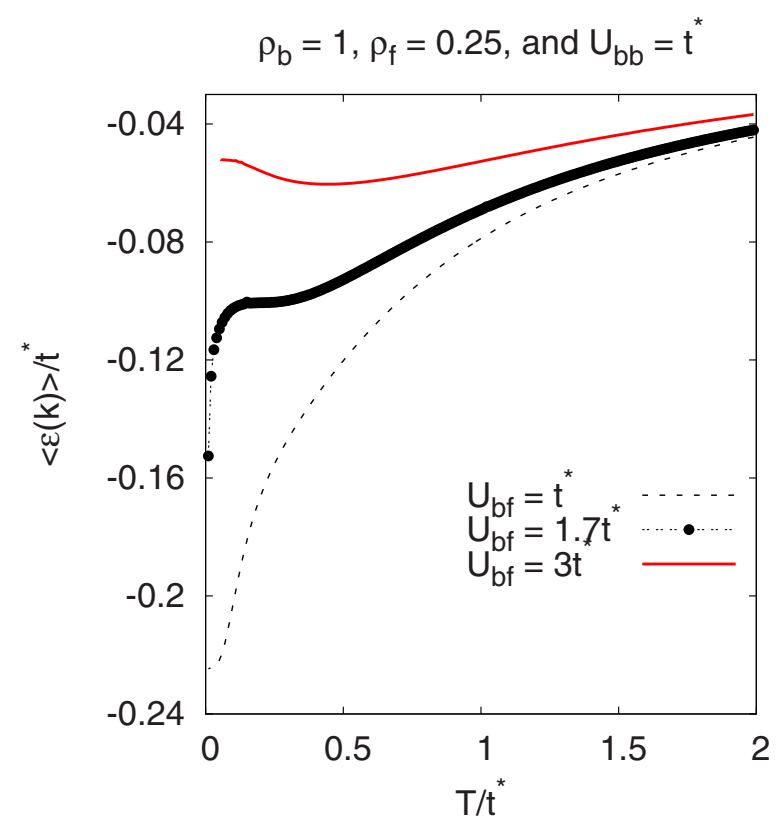

FIG. 4. (Color online) The average kinetic energy of the fermions $\langle\epsilon(\mathbf{k})\rangle$ is shown as a function of temperature $T$.

$U_{b f}=3 t^{*}$. The nonmonotonic behavior occurs only for large enough $U_{b f}$ values and is a consequence of the correlation induced band gaps that are present in the many-body DOS, as discussed in Sec. IV B. This may be one of the most direct ways, with current available technology, to infer the changes in the DOS as a function of $T$ in experiment.

\section{CONCLUSIONS}

In this work, we analyzed Fermi-Bose mixtures consisting of light fermions and heavy bosons that are loaded into optical lattices. To describe such mixtures, we considered the Fermi-Bose version of the FK model, the Fermi-Fermi version of which has been widely discussed in the condensedmatter literature. In our model, we assumed that the bosons are localized such that their tunneling to other sites vanishes $\left(t_{b}=0\right)$ but that the system can statistically sample all low energy configurations of the heavy atoms. This perspective makes sense in ultracold atomic experiments if the difference in the tunneling amplitudes between the light fermions and heavy bosons is large enough so that the quantum nature of the bosons can be neglected, but they can reorganize their positions to allow the system to sample different configurations [31]. An alternative perspective is that once the optical lattice has been introduced, the heavy bosons become frozen into a specific configuration, that is randomly chosen from the configurations that are energetically favorable in the statistical-mechanical ensemble; as one repeats many experiments and averages over the different configurations, one would then reproduce the results of the FK model described here [32].

First, we discussed the symmetries of the Hamiltonian, and showed that the Fermi-Bose FK model can be mapped exactly onto the spinless Fermi-Fermi FK model at zero temperature for all parameter space as long as the mixture is 
thermodynamically stable. Since this mapping is only approximate at low temperatures and fails at high temperatures, we developed DMFT to investigate the effects of temperature (recall that the DMFT becomes exact in infinite dimensions). We also calculated spectral moment sum rules for the retarded Green's function and retarded self-energy, and used them to check the accuracy of our numerical calculations, as well as to reduce the computational cost. When bosons and fermions are uncoupled $\left(U_{b f}=0\right)$, we showed that, as the boson filling increases, the mapping is possible only for lower $T$ values at a given $U_{b b}$, and is possible only for lower $U_{b b}$ values at given $T$. This is because it is energetically more favorable to have occupation of multiple $n_{b}$ states as a function of increasing $T$ and/or decreasing $U_{b b}$ due to the Bose statistics. As $U_{b f}$ increases, we found that the mapping is possible for smaller and smaller parameter space compared to the $U_{b f}=0$ limit. This is because the coupling between bosons and fermions induces an attractive interaction between bosons such that the effective boson-boson repulsion $U_{b b}^{\text {eff }}$ decreases by some amount that is proportional to $U_{b f}^{2}$ to the lowest order in $U_{b f}$. Therefore, increasing $U_{b f}$ leads to an occupation of additional $n_{b}$ states.

We also presented typical numerical results for the FermiBose FK model including the occupancy of bosonic states, single-particle many-body DOS for the fermions, experimentally relevant momentum distribution, and the average kinetic energy. We found that the occupancy of multiple bosonic states has a strong effect on the DOS for the fermions, leading to strong modulations as a function of frequency. The number of peaks corresponds to the number of bosonic states that are occupied, and it increases as a func- tion of increasing $T$ and/or $U_{b f}$. In addition, we showed that increasing $U_{b f}$ at a fixed $T$ broadens the momentum distribution of the fermions, just like the effects of temperature by itself. We also showed how the average kinetic energy evolves with $T$ and how one can infer changes to the DOS via structure in the average kinetic energy.

We hope that some of these results could be experimentally realized in ultracold atomic systems. We think, for instance, $\mathrm{K}-\mathrm{Rb}$, Li-K, or Li-Cs mixtures are a good initial candidates for simulating the Bose-Fermi FK model. In addition, one could also create species-dependent optical lattices for different isotopes of the same atom such that the bosonic isotope is localized but not the fermionic one.

Finally, if one recalls the local-density approximation as a first approximation to the effects of the trap in a real experimental system, we expect that the methods described here could be quickly used to generate approximate results for density distributions across the trap and for a variation of the density of states or of the momentum distribution. Such results are beyond the scope of this work, but could be investigated if one is interested in directly modeling a specific experiment that is described by the Fermi-Bose FK model.

\section{ACKNOWLEDGMENTS}

This work was partially completed during M.I.'s stay at the Joint Quantum Institute (National Institute of Standards and Technology and University of Maryland). J.K.F. acknowledges support under ARO Grant No. W911NF0710576 with funds from the DARPA OLE Program. Part of this work was completed during a stay at the Aspen Center for Physics.
[1] H. Heiselberg, C. J. Pethick, H. Smith, and L. Viverit, Phys. Rev. Lett. 85, 2418 (2000); F. Matera, Phys. Rev. A 68, 043624 (2003); S. Powell, S. Sachdev, and H.-P. Büchler, Phys. Rev. B 72, 024534 (2005); I. Titvinidze, M. Snoek, and W. Hofstetter, ibid. 79, 144506 (2009).

[2] J. K. Freericks, M. M. Maśka, Anzi Hu, T. M. Hanna, C. J. Williams, P. S. Julienne, and R. Lemański, e-print arXiv:0908.1794.

[3] M. P. A. Fisher, P. B. Weichman, G. Grinstein, and D. S. Fisher, Phys. Rev. B 40, 546 (1989).

[4] K. Günter, T. Stöferle, H. Moritz, M. Köhl, and T. Esslinger, Phys. Rev. Lett. 96, 180402 (2006).

[5] S. Ospelkaus, C. Ospelkaus, O. Wille, M. Succo, P. Ernst, K. Sengstock, and K. Bongs, Phys. Rev. Lett. 96, 180403 (2006).

[6] Th. Best, S. Will, U. Schneider, L. Hackermüller, D. van Oosten, I. Bloch, and D.-S. Lühmann, Phys. Rev. Lett. 102, 030408 (2009).

[7] M. Cramer, S. Ospelkaus, C. Ospelkaus, K. Bongs, K. Sengstock, and J. Eisert, Phys. Rev. Lett. 100, 140409 (2008).

[8] D.-S. Lühmann, K. Bongs, K. Sengstock, and D. Pfannkuche, Phys. Rev. Lett. 101, 050402 (2008).

[9] L. Pollet, C. Kollath, U. Schöllwock, and M. Troyer, Phys. Rev. A 77, 023608 (2008).

[10] G. Refael and E. Demler, Phys. Rev. B 77, 144511 (2008).
[11] S. Tewari, R. M. Lutchyn, and S. Das Sarma, Phys. Rev. B 80, 054511 (2009).

[12] If $t_{b}$ is an order of magnitude smaller than $t_{f}$, we expect the quantum effects of the bosons to be an order of magnitude lower than of the fermions. We feel this is an appropriate rough criterion for when applying this model, as the quantum effects of the bosons are sharply reduced relative to that of the fermions.

[13] For the K-Rb system, detailed calculations show that once the lattice depth is smaller than about 8 recoil energies $(\mathrm{Rb})$, one has the $\mathrm{Rb}$ hopping an order of magnitude smaller than the $\mathrm{K}$ hopping [2].

[14] L. M. Falicov and J. C. Kimball, Phys. Rev. Lett. 22, 997 (1969).

[15] C. Ates and K. Ziegler, Phys. Rev. A 71, 063610 (2005).

[16] K. Byczuk and D. Vollhardt, Phys. Rev. B 77, 235106 (2008); Ann. Phys. 18, 622 (2009).

[17] Ch. Gruber and N. Macris, Helv. Phys. Acta 69, 850 (1996); J. Jedrzejewski and R. Lemański, Acta Phys. Pol. B 32, 3243 (2001).

[18] J. K. Freericks and V. Zlatić, Rev. Mod. Phys. 75, 1333 (2003).

[19] U. Brandt and C. Mielsch, Z. Phys. B 75, 365 (1989).

[20] A. B. Harris and R. V. Lange, Phys. Rev. 157, 295 (1967). 
[21] V. M. Turkowski and J. K. Freericks, Phys. Rev. B 73, 075108 (2006); 73, 209902(E) (2006).

[22] V. Turkowski and J. K. Freericks, Phys. Rev. B 77, 205102 (2008).

[23] J. K. Freericks and V. Turkowski, Phys. Rev. B 80, 115119 (2009).

[24] J. K. Freericks, E. H. Lieb, and D. Ueltschi, Phys. Rev. Lett. 88, 106401 (2002); Commun. Math. Phys. 227, 243 (2002).

[25] P. W. Anderson, J. Phys. Chem. Solids 11, 26 (1959).

[26] A. J. Millis, P. B. Littlewood, and B. I. Shraiman, Phys. Rev. Lett. 74, 5144 (1995); A. J. Millis, R. Mueller, and B. I. Shraiman, Phys. Rev. B 54, 5389 (1996).

[27] W. Metzner and D. Vollhardt, Phys. Rev. Lett. 62, 324 (1989).

[28] M. Jarrell, Phys. Rev. Lett. 69, 168 (1992).

[29] D. O. Demchenko, A. V. Joura, and J. K. Freericks, Phys. Rev. Lett. 92, 216401 (2004).

[30] J. T. Stewart, J. P. Gaebler, and D. S. Jin, Nature (London) 454, 744 (2008).

[31] It is difficult to estimate the time scale over which this aver- aging occurs because there is a thermalizationlike process that requires one to understand the nonequilibrium dynamics. As a rough estimate, one can take $t_{b}$ of the bosons multiplied by their number to estimate the number of possible hops of the bosons in a given time interval. If we assume $t_{b}$ is on the order of one-tenth of $t_{f}$, one would expect the thermalization time for the bosons to be about an order of magnitude or so longer. If we believe the fermions thermalize within the optical lattice in the tens to hundreds of ms time scale, then the bosons should do the same in a hundreds of ms to $1 \mathrm{~s}$ time frame, which may be adequate for current experiments which can have hold times in the few seconds range on an optical lattice. Basically, if one believes the fermions have thermalized and are represented by an equilibrium system, than the bosons will do the same in a proportionally longer time as determined by $t_{f} / t_{b}$.

[32] M. M. Maśka, R. Lemański, J. K. Freericks, and C. J. Williams, Phys. Rev. Lett. 101, 060404 (2008). 